\title{
Mechanisms of Photo Double Ionization of Helium by 530 eV Photons
}

\author{
A. Knapp, ${ }^{1}$ A. Kheifets, ${ }^{2}$ I. Bray, ${ }^{3}$ Th. Weber, ${ }^{1}$ A. L. Landers, ${ }^{4}$ S. Schössler, ${ }^{1}$ T. Jahnke, ${ }^{1}$ J. Nickles, ${ }^{1}$ \\ S. Kammer, ${ }^{1}$ O. Jagutzki, ${ }^{1}$ L. Ph. H. Schmidt, ${ }^{1}$ T. Osipov, ${ }^{5}$ J. Rösch ${ }^{6,1}$ M. H. Prior, ${ }^{6}$ \\ H. Schmidt-Böcking, ${ }^{1}$ C. L. Cocke, ${ }^{5}$ and R. Dörner ${ }^{1, *}$ \\ ${ }^{1}$ Institut für Kernphysik, Universität Frankfurt, August-Euler-Strasse 6, D-60486 Frankfurt, Germany \\ ${ }^{2}$ Research School of Physical Sciences and Engineering, Australian National University Canberra ACT 0200, Australia \\ ${ }^{3}$ Centre for Atomic, Molecular and Surface Physics, Murdoch University, Perth, 6150 Australia \\ ${ }^{4}$ Department of Physics, Western Michigan University, Kalamazoo, Michigan 49008 \\ ${ }^{5}$ Department of Physics, Kansas State University, Cardwell Hall, Manhattan, Kansas 66506 \\ ${ }^{6}$ Lawrence Berkeley National Laboratory, Berkeley, California 94720
}

(Received 30 November 2001; published 27 June 2002)

\begin{abstract}
We have measured fully differential cross sections for photo double ionization of helium $450 \mathrm{eV}$ above the threshold. We have found an extremely asymmetric energy sharing between the photoelectrons and an angular asymmetry parameter $\beta \simeq 2$ and $\beta \simeq 0$ for the fast and slow electrons, respectively. The electron angular distributions show a dominance of the shakeoff for $2 \mathrm{eV}$ electrons and clear evidence of an inelastic electron-electron scattering at an electron energy of $30 \mathrm{eV}$. The data are in excellent agreement with convergent close-coupling calculations.
\end{abstract}

DOI: 10.1103/PhysRevLett.89.033004

PACS numbers: $32.80 . \mathrm{Fb}$

How does a single photon couple to two electrons in an atom? This question has been extensively discussed in the literature. Most of this discussion has been focused on the photo double ionization (PDI) of the helium atom which is the simplest two-electron-single-photon process (see McGuire et al. [1] and Briggs and Schmidt [2] for reviews). It is generally believed that at high photon energies, PDI is mediated predominantly via initial state correlation. After a sudden removal of one atomic electron the correlated initial state relaxes onto the new $\mathrm{He}^{+}$eigenstates. This process has been termed shakeoff. In the high photon energy limit, the ratio of the total double to total single ionization cross sections converges to a constant $R=1.67 \%$, a limit expected for the shakeoff $[3,4]$. At low photon energies it has been argued by Samson [5] that one electron absorbs the photon and knocks out the second electron in an $(e, 2 e)$-like collision. This final state correlation process is called in the literature two-step-one, or TS1. The whole discussion on the different types of correlation of the PDI is based solely on theory [6-9] and on measured total cross sections [5].

Detailed experimental and theoretical studies of the angular and energy correlation between the two photoelectrons or, equivalently, the photoelectron and the recoiling ion are presently available in the form of the fully resolved triple differential cross section (TDCS) (see Briggs and Schmidt [2] for a recent review). However, these studies are limited to relatively low photon energies where shakeoff is believed to be not significant. Additionally, in this regime the angular distributions and the energy sharing are determined almost entirely by the long range Coulomb repulsion of the photoelectrons and the dipole selection rules, which completely mask the signatures of particular ionization mechanisms.
This Letter presents experimental data and theoretical calculations of PDI of helium at $530 \mathrm{eV}$ photon energy, where the shakeoff yields a significant contribution. We show that characteristics of the shakeoff and TS1 can be clearly seen in the TDCS. Electrons with an energy in the range of $2 \mathrm{eV}$ are mainly produced by the shakeoff while at $30 \mathrm{eV}$ we find clear evidence of TS1. This confirms a theoretical prediction of Teng and Shakeshaft [9] who found that, at high photon energies, shakeoff and TS1 would leave clear signatures in the electron angular distribution. As we will show below, the virtue of such a study at high photon energies is that the two photoelectrons typically have very different energies and angular distributions, allowing experimental selection of the primary high energy electron which is coupled to the photon.

The experiment has been performed using the COLTRIMS technique (see [10] for a general review and [11,12] for application to synchrotron radiation). The photon beam $(\hbar \omega=529 \mathrm{eV})$ from beam line 4 of the Advanced Light Source (ALS) at the Lawrence Berkeley National Laboratory [13] is focused into a supersonic helium gas jet. Electrons of energy below $60 \mathrm{eV}$ are collected by a combination of electric and magnetic fields onto a large area position sensitive channel plate detector [14]. From the time of flight and the position of impact the momentum vector of the electron is deduced [15]. The electric field guides the ions with $4 \pi$ collection solid angle for all momenta onto a second position sensitive detector. The ion charge state and momentum vector are again obtained from the time of flight and position of impact. The momentum vector of the fast electron is calculated from the measured slow electron and recoiling ion using momentum conservation. 
We corroborate our experimental findings by performing a series of convergent close-coupling (CCC) calculations (see Kheifets and Bray [16] for details). In brief, the fast photoelectron of energy $E_{1}$ is described in the CCC model by a Coulomb wave, whereas the slow photoelectron of energy $E_{2}$ is represented by a positive energy pseudostate of the $\mathrm{He}^{+}$ion. The shakeoff is reproduced in the model by the dipole matrix element between a highly correlated ground state wave function and a product of the Coulomb wave with energy $E_{1}$ and the pseudostate with energy $E_{2}$. The TS1 is represented by the inelastic scattering of the fast electron on an eigen- or pseudostate of the ion. The amplitude of this process is calculated as a nondiagonal element of the scattering $T$ matrix. The diagonal part of the $T$ matrix describes the elastic electron scattering in which the quantum state of the slow electron does not change. The only effect of this elastic scattering is the distortion of the Coulomb wave representing the fast electron, and so is attributed to the shakeoff [7]. The nondiagonal part of the $T$ matrix can be turned off at will, thus simulating the shakeoff only PDI process. By retaining both the diagonal and nondiagonal parts of the $T$ matrix we perform a standard CCC calculation in which both the shakeoff and the TS1 are taken into account. This calculation is not any different from other CCC calculations performed at much lower photon energies.

We calculate a succession of cross sections starting from the fully resolved TDCS $d^{3} \sigma /\left(d \Omega_{1} d \Omega_{2} d E_{1}\right)$. Integrating the TDCS over $d \Omega_{2}$ reduces it to the double differential cross section (DDCS) which determines the energy and angular distribution of one photoelectron integrated over all angles of the second electron. Within the dipole approximation the DDCS is given by [17]

$$
\frac{d^{2} \sigma}{d \Omega d E}=\frac{d \sigma}{d E} \frac{1}{4 \pi}\left[1+\beta(E)\left(\frac{3}{2} \cos ^{2} \vartheta-\frac{1}{2}\right)\right] .
$$

Here $d \sigma / d E$ is the single differential cross section (SDCS) which gives the energy sharing distribution between the photoelectrons, $\beta$ is the angular asymmetry parameter, and $\vartheta$ is the polar angle of the electron with respect to the polarization axis of light.

The top panel of Fig. 1 shows the measured and calculated SDCS. It has a characteristic U shape and peaks sharply at 0 and $450 \mathrm{eV}$. This trend is very well represented by the CCC calculation and has already been established in earlier calculations $[9,18,19]$. This shape of the curve is in contrast to the SDCS close to the threshold which is almost flat.

The bottom panel of Fig. 1 shows the measured and calculated $\beta$ parameter. Note that at high incident energies, as is the present case, the CCC-calculated SDCS or $\beta$ parameter cannot be readily determined with sufficient accuracy away from highly asymmetric energy sharing conditions. The SDCS can be extrapolated over the whole energy range by knowing the accurate total PDI cross sec-

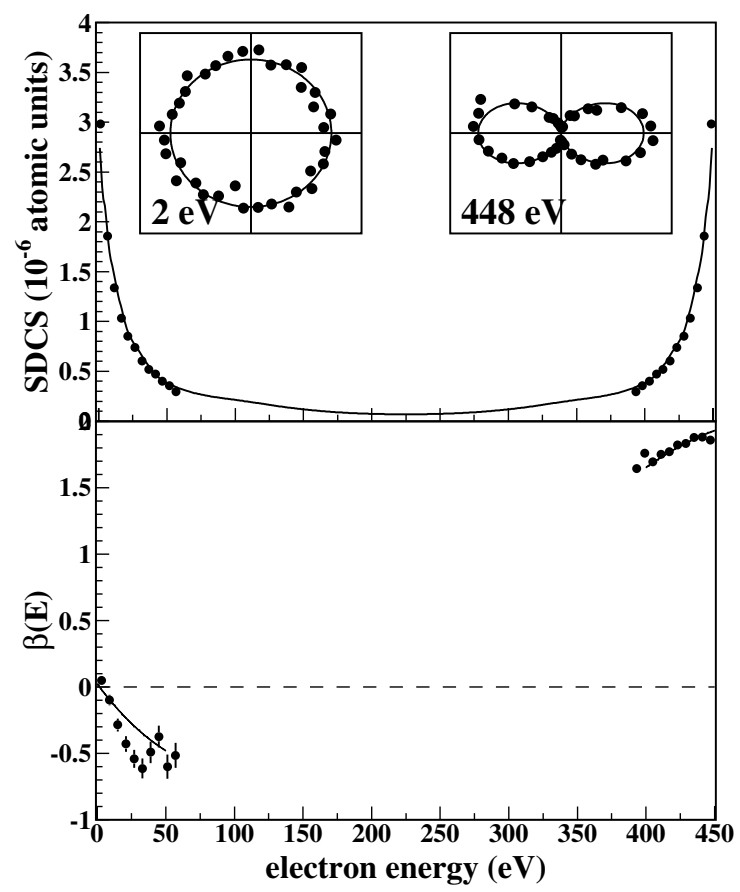

FIG. 1. PDI of $\mathrm{He}$ at $\hbar \omega=529 \mathrm{eV}$. (a) SDCS $d \sigma / d E$. The line is the CCC calculation. The insets show the DDCS $d \sigma^{2} /(d \Omega d E)$ at $E=2$ and $448 \mathrm{eV}$ (the vertical axis is the light propagation); the line is obtained using Eq. (1) (see text). The experimental data are normalized to the CCC calculation. (Bottom panel) The asymmetry parameter $\beta$ versus the electron energy.

tion $\sigma=\int_{0}^{E / 2}(d \sigma / d E) d E$. A reliable calculation of the $\beta$ parameter exists only at $E_{2} \leq 50 \mathrm{eV}$ and $E_{1} \geq 400 \mathrm{eV}$, as shown in Fig. 1.

We verified the validity of the dipole approximation for the DDCS of Eq. (1) and did not find, within the statistical uncertainty, any significant forward-to-backward asymmetry in our data. Two examples of the experimental DDCS at $E=2$ and $448 \mathrm{eV}$ are shown in the insets together with the line obtained from Eq. (1), using CCC estimates of the SDCS and $\beta$. A very asymmetric energy sharing together with an angular asymmetry parameter $\beta \simeq 2$ indicate that the fast electron absorbs not only most of the photon energy but also its angular momentum. This directly suggests an interpretation of the PDI as a two-step process with the fast electron being the primary photoelectron. The slow electron is isotropic at very low energy, as expected for the shakeoff, while $\beta$ becomes slightly negative for higher energies. As we will show below in more detail, this slightly preferred emission perpendicular to the polarization is a consequence of a binary encounter between the two electrons.

To learn more about the type of correlation (initial or final state) and the corresponding mechanism (shakeoff or TS1) by which the second electron is emitted an overview of the TDCS of both electrons is given in Fig. 2. The horizontal axis shows the polar angle $\vartheta_{1}$ of the fast electron with respect to the polarization; the vertical axis displays 


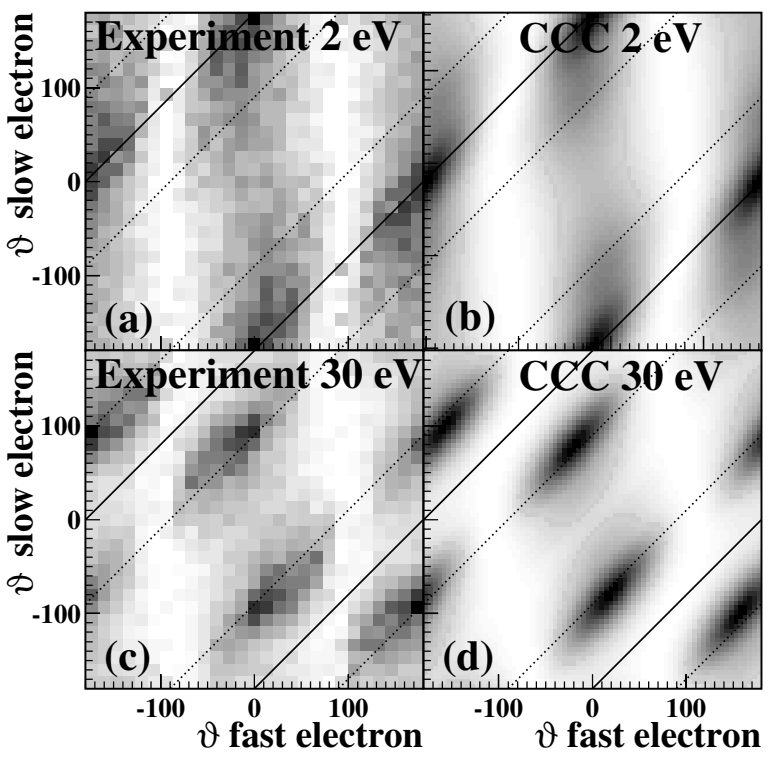

FIG. 2. Overview of the TDCS at $450 \mathrm{eV}$ excess energy [(a), (c) experiment, (b),(d) CCC calculation] and coplanar emission for electron energies between $0-3 \mathrm{eV}$ for the slow electrons (447-450 eV for the fast electrons) (a),(b) and $20-40 \mathrm{eV}$ $(410-430 \mathrm{eV})(\mathrm{c}),(\mathrm{d})$. The horizontal axis shows the angle $\vartheta_{1}$ of the fast electron with respect to the polarization vector; the vertical axis displays the angle $\vartheta_{2}$ of the slow electron. The solid lines indicate the back-to-back emission $\vartheta_{12}=180^{\circ}$ (the shakeoff) and the dashed lines define emission of the two electrons of an angle $\vartheta_{12}=90^{\circ}$, as expected from the TS1. Experimental data and theory are integrated over the same energy and angular ranges.

the angle of the slow electron $\vartheta_{2}$. The electrons and polarization are chosen to be coplanar, i.e., the slow electron is within $\pm 35^{\circ}$ in the plane defined by the fast electron and the polarization axis. The fast electron has almost no intensity at $\vartheta_{1}=90^{\circ}$ reflecting a $\beta$ parameter of close to 2 [see Eq. (1)]. The two left panels show the experimental data, whereas the corresponding right panels exhibit the TDCS from the CCC calculations. Good agreement between theory and experiment can be seen for all angles at both energy sharings.

In these two-dimensional plots the typical characteristics of the shakeoff and TS1 can be clearly identified. For the shakeoff, one would expect that the slow electron is emitted isotropically or slightly backwards to the fast primary electron $[9,20]$. The locus of such events is indicated by the solid lines $\vartheta_{12} \equiv\left|\vartheta_{1}-\vartheta_{2}\right|=180^{\circ}$. The TS1 is, in contrast, a binary encounter between particles of equal mass; hence one expects it to peak at $\vartheta_{12}=90^{\circ}$. This is indicated by the dashed lines also in Fig. 2. At $E_{2}=2 \mathrm{eV}$ the maximum of the TDCS follows closely the $\vartheta_{12}=180^{\circ}$ lines, supporting that such slow electrons are produced predominantly via shakeoff. At $E_{2}=30 \mathrm{eV}$ the maxima are clearly along the lines with $\vartheta_{12}=90^{\circ}$, indicating a switch from the shakeoff to a binary collision. A significant energy transfer from the primary to the secondary electron seems to require a binary collision and is not likely via the shakeoff. It can be noted from the U-shaped SDCS (Fig. 1) that the contribution of the slow shakeoff electrons to the total cross section is by far dominant over the electrons of $30 \mathrm{eV}$ and higher. Thus the total PDI cross section is dominated by the shakeoff process [7].

For a closer inspection and a detailed comparison with theory we have plotted a small subset of the data shown in Fig. 2 as polar plots (Fig. 3). In all cases, one of the electrons has been fixed to one direction within $10^{\circ}$ of the linear polarization, and the TDCS of the complementary electron is plotted. Thus data from Fig. 2 within the range $-10^{\circ}<$ $\vartheta_{1}<10^{\circ}$ appear in Figs. 3(b) and 3(d), and data in the range $-10^{\circ}<\vartheta_{2}<10^{\circ}$ are shown in Figs. 3(a) and 3(c).

The TDCS for electrons $E_{2}<3 \mathrm{eV}$ [Fig. 3(b)] has a pearlike shape peaked at $180^{\circ}$ to the fast electron. Contrary to all TDCS reported at lower photon energies so far, these slow electrons show a significant intensity for parallel emission in the same direction. This is possible because of the very asymmetric energy sharing of the two electrons. The solid line is a full CCC calculation which is in excellent agreement with the measurements. The dashed line is the CCC calculation representing the shakeoff in which only the diagonal part of the $T$ matrix is retained. The

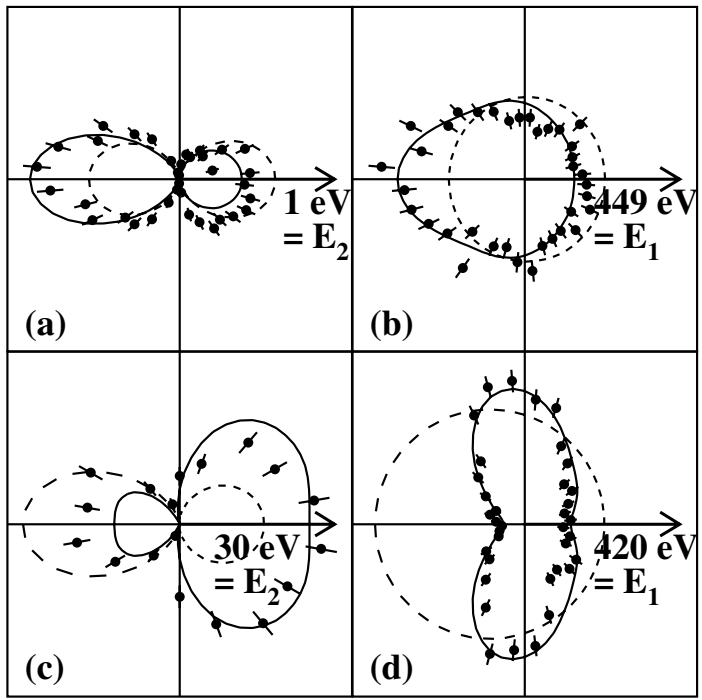

FIG. 3. TDCS of the He PDI at $529 \mathrm{eV}$ photon energy. In all panels the electrons are coplanar within $\pm 25^{\circ}$; the polarization axis (of linearly polarized light) is horizontal. The direction and the energy of one of the two electrons is fixed, as indicated by the number and the arrow, i.e., the slow electron is fixed in (a) and (c) and the fast electron is fixed in (b) and (d). The polar plots show the angular distribution of the complementary electron. The upper panels (a) and (b) are for the case $E_{2} \simeq$ $2 \mathrm{eV}$; the lower panels (c) and (d) are for $E_{2} \simeq 30 \mathrm{eV}$. The solid lines are a full CCC calculation and the dashed lines are a shakeoff only $\mathrm{CCC}$ calculation. The measurements are normalized to the full CCC calculation. The shakeoff calculation in (c) is multiplied by 0.4 . The measurements and calculations are integrated over the same angular and energy ranges. (a) $447<E_{1}<450 \mathrm{eV},-10^{\circ}<\vartheta_{1}<10^{\circ}$; (b) $0<E_{2}<$ $3 \mathrm{eV},-10^{\circ}<\vartheta_{2}<10^{\circ}$; (c) $410<E_{1}<430 \mathrm{eV},-10^{\circ}<$ $\vartheta_{1}<10^{\circ}$; (d) $20<E_{2}<40 \mathrm{eV},-10^{\circ}<\vartheta_{2}<10^{\circ}$. 
corresponding TDCS of the fast electron $\left(E_{1}>447 \mathrm{eV}\right)$ shows a dipolar shape [Fig. 3(a)] with the lobe for parallel emission in the same direction of the electrons being slightly suppressed for the full calculation. Again the full $\mathrm{CCC}$ calculation is in excellent agreement with the experimental data.

The TDCSs for electrons $E_{2} \simeq 30 \mathrm{eV}$ [Figs. 3(c) and $3(\mathrm{~d})]$ are completely different from the low energy ones. We find emission of the electron into a narrow cone at $90^{\circ}$ to the fast electron [Fig. 3(d)]. An angle of $90^{\circ}$ between the electrons is expected from a binary collision between the electrons. Again the full CCC calculation is in very good agreement with the measurements. The shape of the shakeoff only calculation is in complete disagreement with the data; the overall size, however, is comparable. The complete CCC calculation is a coherent sum of the shakeoff and the TS1 contribution. Since the fast electron peaks parallel to the polarization, the $90^{\circ}$ angle between the electrons also leads to a slightly negative $\beta$ at these electron energies [see also Fig. 1 (bottom panel)]. Figure 3(c) shows the inverse energy sharing to Fig. 3(d), i.e., the data points show the fast electron distribution; the slow $(30 \mathrm{eV})$ electron is selected along the polarization. In this situation again the angular distribution of the fast electron shows an almost dipolar pattern imprinted by the photon. Since this configuration is not accessible by a binary collision the cross section is very small.

In conclusion, we have presented experimental and theoretical TDCS of the PDI of helium at the photon energy $\hbar \omega=529 \mathrm{eV}$ (excess energy of $450 \mathrm{eV}$ above the double ionization threshold). At such a high excess energy, with highly asymmetric kinematics, we may think of the fast and slow electrons as being distinguishable. The angular distribution between the two electrons indicates that the very low energy secondary electrons are mostly emitted via the shakeoff process while higher energy transfer requires a hard binary collision and leads to an angle of $90^{\circ}$ between the electrons.

This work was supported in part by BMBF, DFG, and the Chemical Sciences, Geosciences and Biosciences Division, Office of Basic Energy Sciences, Office of Science, U.S. Department of Energy. The computations were performed at the National Facility of the Australian Partnership for Advanced Computing. R. D. was supported by the
Heisenberg Programm der DFG. A. K. and Th. W. thank Graduiertenförderung des Landes Hessen for financial support. Many thanks to E. Arenholz and T. Young and the staff of the ALS for their extraordinary support during our beam time. R. D. acknowledges many enlightening discussions with J. Berakdar, A. Becker, and S. Keller.

*Electronic address: doerner@hsb.uni-frankfurt.de

[1] J. McGuire, N. Berrah, R. Bartlett, J. Samson, J. Tanis, C. Cocke, and A. Schlachter, J. Phys. B 28, 913 (1995).

[2] J. Briggs and V. Schmidt, J. Phys. B 33, R1 (2000).

[3] J. Levin, D. Lindle, N. Keller, R. Miller, Y. Azuma, N. Mansour, H. Berry, and I. Sellin, Phys. Rev. Lett. 67, 968 (1991).

[4] L. Spielberger et al., Phys. Rev. Lett. 74, 4615 (1995).

[5] J. Samson, Phys. Rev. Lett. 65, 2861 (1990).

[6] K. Hino, T. Ishihara, F. Shimizu, N. Toshima, and J. H. McGuire, Phys. Rev. A 48, 1271 (1993).

[7] A. Kheifets, J. Phys. B 34, L247 (2001).

[8] S. Keller, J. Phys. B 33, L513 (2000).

[9] Z. Teng and R. Shakeshaft, Phys. Rev. A 49, 3597 (1994).

[10] R. Dörner, V. Mergel, O. Jagutzki, L. Spielberger, J. Ullrich, R. Moshammer, and H. Schmidt-Böcking, Phys. Rep. 330, 96 (2000).

[11] R. Dörner et al., Phys. Rev. Lett. 77, 1024 (1996); see also erratum in Phys. Rev. Lett. 78, 2031(E) (1997).

[12] R. Dörner et al., Phys. Rev. A 57, 1074 (1998).

[13] A. T. Young, J. Feng, E. Arenholz, H. A. Padmore, T. Henderson, S. Marks, E. Hoyer, R. Schlueter, J. B. Kortright, V. Martynov, C. Steier, and G. Portmann, Nucl. Instrum. Methods Phys. Res., Sect. A 467-468, 549 (2001).

[14] (See Roentdek.com for details of the detectors.)

[15] R. Moshammer, J. Ullrich, M. Unverzagt, W. Schmitt, P. Jardin, R. Olson, R. Dörner, V. Mergel, and H. SchmidtBöcking, Nucl. Instrum. Methods Phys. Res., Sect. B 107, 62 (1996).

[16] A. S. Kheifets and I. Bray, J. Phys. B 31, L447 (1998).

[17] M. Y. Amusia, Atomic Photoeffect (Plenum Press, New York, 1990).

[18] D. Proulx and R. Shakeshaft, Phys. Rev. A 48, R875 (1993).

[19] M. Kornberg and J. Miraglia, Phys. Rev. A 48, 3714 (1993).

[20] F. Maulbetsch and J. Briggs, J. Phys. B 27, 4095 (1994). 